\title{
IoT Enabled Air Quality Monitoring for Health- Aware Commuting Recommendation in Smart Cities
}

\author{
Riaz UlAmin ${ }^{1} *$ \\ Dept. of CS, University of Okara \\ Okara, Pakistan
}

\author{
Muhammad Akram² \\ Dept. of Computer Engineering \\ BUITEMS, Quetta, Pakistan
}

\author{
Najeeb Ullah ${ }^{3}$ \\ Dept. of Telecom Engineering \\ BUITEMS, Quetta, Pakistan \\ Muhammad Ashraf ${ }^{4}$ \\ Dept. of Software Engineering \\ BUITEMS, Quetta, Pakistan
}

\author{
Abdul Sattar Malik ${ }^{5}$ \\ Dept. of Electrical Engineering \\ BZU, Multan, Pakistan
}

\begin{abstract}
The importance of air pollution control in smart cities has been realized by almost every department of society. Research community has been working in collaboration with industry to craft sensors for measuring different types of pollution levels in the environment. However, it is rarely possible to implant the sensors in all geographical areas. It is important to measure pollution levels in almost every part of the world with life and implement clean environment policies. However, in unplanned areas, the implementation of environmental policies faces problems because such areas lack in communication infrastructure and cost of huge amount of fixed or static sensors. This work envisions availability of sensor-equipped-VANET based system to monitor pollution levels in different areas of an unplanned city. This paper proposes an autonomous VANET system that can carry environmental sensors to collect data from an area at different intervals, process it to transform data into information and forward the information to node that has capability to collect all information and then send it to server machine for further process either using VANET or some reliable network connectivity. Based on the collected data, this research further contributes health aware commuting recommendation based on cost effective monitoring of air quality.
\end{abstract}

Keywords-Un-planned Areas; Vehicular ad hoc Networks; Pollution Monitoring; AQI; Health Aware Commuting Recommendations

\section{INTRODUCTION}

VANET are networks that can be characterized by their openness in nature, uninterrupted energy availability and high speed mobility. Research on vehicular communication is continuously emerging and evolving. It not only addresses safety of life aspects on the road but it also considers non safety related applications. Classifications of such applications can easily be found in literature [1]. One important application of VANET proposed in this paper is to monitor air pollution particularly in unplanned areas. Unplanned areas are defined as the areas that are attributed by several characteristics. Usually such areas are developed without proper town planning regulations. Thus such areas, in essence, lack in availability of different services and communication infrastructures. Lack of town planning and industrial zone planning not only poses threats to clean environment but also makes conventional air pollution monitoring systems harder to implement. Particularly, in developing countries, with growth in economy, unplanned land development is likely to take place. Growth in industrialization and unplanned land development poses several challenges to the environment. Continuous monitoring of different areas for clean environmental measurements is already a challenge, furthermore, lack of communication infrastructure for environmental data collection and higher cost of large scale fixed sensor network for environment monitoring system, extravagate the problem. On the other hand it is important to monitor such areas for air pollution. This work exploits VANET services as cost-effective means to monitor air pollution of such unplanned areas.

\section{A. Vehicular Ad -hoc Network (VANET) and Wireless Sensor Networks (WSN)}

VANET are a subclass of MANET that can be equipped with several types of sensors. Equipping vehicles with such sensors help to envision several applications that can be deployed over VANET. One such application introduced in this paper, serves monitoring an unplanned area for air pollution. Importance of air pollution monitoring has been widely realized in society. Electronics engineering research and industrial community has been successfully crafting wide range of sensors to measure different types of air pollutions. The deployment of theses sensors can greatly affect the monitoring system's performance. Majorly the deployment of sensors to for monitoring an area for air pollution can be executed in one of the following three approaches.

1) Static deployment of sensors, the information is periodically collected by these sensors and forwarded to head node using fixed network infrastructure (wired or wireless). The head nodes usually aggregate information, analyze and filters it, and forwards it to decision making node for further process. This approach has several challenges. These challenges include firstly the higher cost for deployment of sensors at large scale area, secondly, energy constrained air may also cause sensor node failures, and lastly but not the least in some cases the objects that needs to be sensed can be

\footnotetext{
*Corresponding Author
} 
mobile in nature, thus static sensors could face limitation in coverage of object.

2) Mobile sensor based deployment. This approach considers to deploy sensors in explicitly mobile mode, no static/fixed sensors are assumed. These sensors can form groups/clusters. Information from mobile sensors is collected by cluster-heads, and then cluster-head after performing initial filtering forwards the data to some server using network based on cellular/ infrastructure-technology. There are several pros and cons of this deployment technique. This technique offers low cost monitoring of an area. It resolves the monitoring of target object mobility however, scale of its deployment and energy of mobile sensor nodes remains questionable.

3) The Hybrid Approach. This approach for deployment of sensors to monitor an area considers use of both static and mobile nodes. Conventional mobile sensor network following this approach of deployment also considers infrastructure based network availability for communication among sensor nodes and for communication required for data collection. This approach has several advantages over the previous two approaches, such as large scale area coverage, design dependent cost and target object mobility tracking, however, this approach is also not without drawbacks. This approach is complex to implement and if the design of network is not efficient, it may cause higher cost. Similarly, energy of sensor nodes remains a challenge.

\section{LITERATURE REVIEW}

There are several contributions in literature that are related to this research. In one of such contribution an online GPRS array for air pollution monitoring is proposed with system that consists of mobile sensing unit, a fixed Internet-Enabled Pollution Monitoring Server (Pollution-Server), sensor array $\left(\mathrm{NO}_{2}, \mathrm{CO} \& \mathrm{SO}_{2}\right)$, GPS and GPRS module, which are integrated with each sensing unit. Sensed data from sensor array with location information are sent to the server through GPRS for further handling and investigation. Public can access the authorized air pollution information by customized Web App. The proposed system was tested in the city of Sharjah UAE [2]. The proposed system has limited scale and scope given the use of fixed infrastructure for collection of sensor data. Another similar work a vehicular pollution monitoring system designed based on internet of things (IoT) capable for monitoring pollutant on city road caused by vehicles emission is proposed in [3]. Sensors of different gases are installed at fixed position on city road. The proposed systems also guarantees the presence of wireless sensors for vehicle pollution system that specify in a straight forward accessibility of real time data through internet using IoT. However this system is installed at fixed position and is able for monitoring pollutant from vehicle emission only. A Mobile Air Quality Monitoring Network (MAQUMON), is proposed in which sensor nodes are mounted on cars. These sensor nodes are consisted of different hardware components including Gas sensors for measuring $\mathrm{CO}$, NO2 and ozone concentration in Air. Data samples from sensor are taken differently for different scenarios. Samples are taken very frequently when car is in motion; while it is taken a few times an hour when a car is parked. These Samples are store and tagged with a time information and location. A Wi-Fi hotspot is used for collection of data, after collection of data it is being processed and published on the Sensor Map portal [4]. The research is closely related to our research in terms of use of VANET for collection of sensed data. However, this research further makes commuting recommendations based on the data collected through sensors. The concept of a Vehicular Wireless Sensor Network (VSN) is proposed in [5] in which sensors are deployed on cars for monitoring air quality. In this framework a is vehicle set with a gas sensor of CO2, a GPS module, a GSM module and ZigBee- based intra- vehicle wireless network. The concentration of $\mathrm{CO} 2$ in air is measured in an area of interest and the sensed data is reported through GSM short messages and collected by a server. The collected data is integrated with Google Maps for the demonstration of result. However the proposed framework uses an expensive GSM short messaging for reporting. Furthermore the recommendations on the basis of data are not in the scope of this research.

Authors in [6] proposed a low cost solar powered air quality monitoring system based on ZigBee wireless network for a fixed station. The proposed prototype was installed at fixed locations at school surrounding. The hardware prototype was consist of set of sensors array for monitoring Carbon monoxide (CO) ,Nitrogen dioxides (NO2), relative humidity and temperature at school surrounding. The design was based on Arduino (UNO) and a ZigBee wireless node. The sensed date was communicated through ZigBee wireless node with low power consumption. The sensed data was monitored on desktop/ laptop through an application designed using lab view. However fixed air quality monitoring station has limitations. Because People's real life exposure to air pollutants is not achieved. At a fixed air quality monitoring stations only the pollutants in air at a fixed locations/area can be monitored, which is inefficient to capture the spatial variability in urban or industrial environment. For this purpose a mobile air quality monitoring system can be used to monitor the air quality which solves the entire existing problem associated with fixed air quality monitoring stations. Authors in another research [7] proposed low-cost air quality system for urban area monitoring through mobile sensing and low cost reliable sensors. A theoretical model is presented which involves two types of mobile sensing network, first is "Personal sensing network" and second is "public transport network". A Personal sensing prototype is designed which measure the concentration of Carbon monoxide (CO) in air. Research proposed two sub models. The first sub model is developed for measuring the concentration of $\mathrm{CO}$ in stationary places and second sub model is designed for measuring the concentration of $\mathrm{CO}$ in mobile situations. The sensed data with time and location information is stored to the flash memory and then uploaded to the local server and results are generated by graphs and heat map. In this paper theoretical model for, personal sensing network and public transport sensing network was proposed. But only one prototype is designed for personal sensing network, which can measure only the concentration of $\mathrm{CO}$ in air. However the proposed "Public transport sensing network" for future work, require more sensing devices for air quality monitoring. 
A low cost, low power outdoor air pollution monitoring system called Gas Mobile was proposed in [8]. The research proposed prototype consists of Ozone (O3) gas sensor and an off-the-shelf smart phone. An USB interface is used to communicate smart phone with sensor module. Sensed data from the sensor module with time and location information, from build in GPS module is uploaded to sever using cellular network. Public can access the official data from modified web and mobile apps. For improving the sensor data quality two methods were proposed and implemented. The proposed prototype was mounted on bicycle (protected from wind) and the measurements were taken from several bicycles across the city. However in the proposed system only one gas sensor is used for measurement of $\mathrm{O} 3$ in atmosphere which is inefficient for measuring over-all air quality. The proposed system is low in data accuracy and reliability as the sensor are mounted on bicycles using handbags, etc. In [9] Authors Proposed Real time Air pollution monitoring system using Mobile phone. In this system the hardware prototype which consist of gas sensors, GPS, Bluetooth modem and a chip microcontroller is installed on the buses to collect the concentration of gases such as $\mathrm{CO}, \mathrm{N}$, smoke and temperature. The sensed data from sensors are sent to the control central unit that uploads this data to Internet through a Google Maps interfaced with Bluetooth module. However the proposed system is costly as the sensed data is simultaneously uploaded to the internet and interfaced with Google map. The proposed system fails when there is no availability of internet.

Researchers proposed air quality monitoring through mobile sensing in metropolitan areas [10]. In the proposed work an MSB (Mobile Sensing Box) was designed. The MSB consist of three main units Sensors, GPS module and a mobile phone. Sensors are used for Data collection, time and location information is carried by GPS module and the transmission of data is done by cellular mobile phone. The prototype is mounted on the car which travels around the city. The sensed data is then uploaded to cloud server. Public can accesses authorized air pollution information through web portal. However the proposed work is costly, because each sensed data is uploaded to cloud server using cellular modem.

\section{SYSTEM DESIGN}

Research presented in this paper considers implanting air monitoring sensors in vehicles. In theory it resembles the approach 2 presented in the previous section, however, it has advantage of no energy limitation of sensors and scale of deployment of sensor nodes. As the area being monitored scales with vehicle's mobility in an area. This approach also permits to collect and share data at cluster head without any fixed network infrastructure. The information collected at different sensor nodes is forwarded to central collection point at cluster head node using VANET communication protocol such as IEEE 802.11p. This approach reduces the cost of network used as IEEE 802.11p is free to use and an efficient communication among vehicle can be executed following this standard.

This hardware prototype shown in Fig. 1 consists of several components. Two gas sensors are connected to Arduino Mega 2560, which measure the concentration level of
$\mathrm{CO}$ and NO2 in air. The Sensors are programmed in a way that they measure the concentration levels of gases with interval of every two seconds. A GPS module is connected to Arduino board which gives information about locations in form of Latitude and Longitude.

For taking time and date information, an RTC module is connected to Arduino board. A storing unit is connected to Arduino board which stores all the data in the form of string, including the data from sensors, GPS and RTC. A XBEE module is connected to Arduino board which transmits the stored data to a server. To minimize the cost, the proposed system considers using storing unit for storing and transmitting data via XBEE module to server. Once data is received at server, it further stores it to a separate database for records. Through designed queries the data can be filtered and sorted for days, months and years. The hardware prototype designed and implemented in this research is portable and can be connected to any vehicle at different positions. In this case, the hardware prototype was connected to vehicles powered by vehicle battery.

Vehicles can have their route spanned over a large area. These areas may be monitored for real time concentration levels of several gases in air. This hardware prototype can also be used as a personal sensing device with addition of on board external battery. The hardware prototype was tested to perform both in stationary and mobile scenarios.

Integration of VANET and wireless sensor networks (WSN) has already been presented in literature. However to best of knowledge, it is first contribution to monitor an unplanned area with the help of VANET based sensor network for air pollution. There are several types of air pollutions, however, this research considered measuring NO2 in the air by using this Air quality measurement module mounted in university buses measuring NO2 in different areas of the city. Fig. 2 shows the general methodology taken in this research.

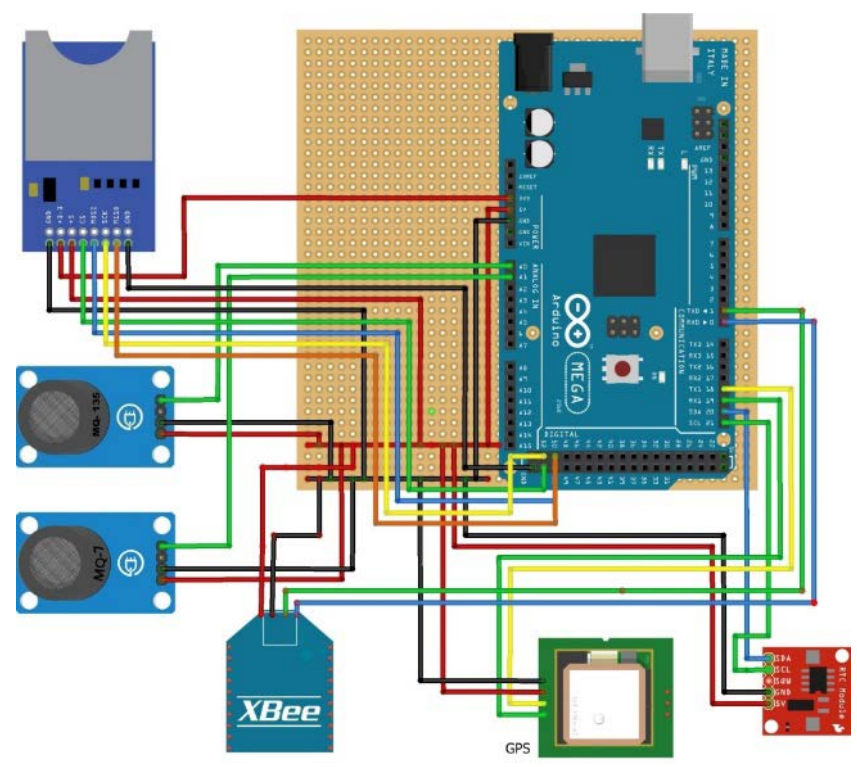

Fig. 1. Block Diagram of Hardware Prototype. 


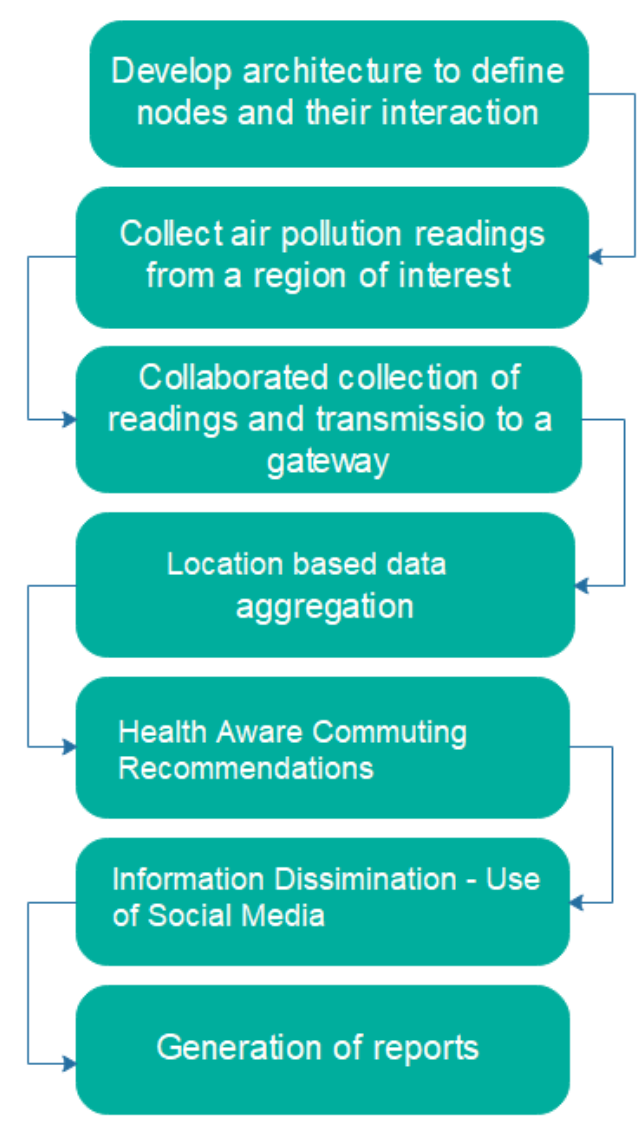

Fig. 2. Block Diagram of System Design.

The data was clustered based on location of measurements. Locations with measurements of NO2 were recorded using GPS module integrated within this prototype Air quality measurement modules. The data was cleansed and clustered using location information. The measurements were further grouped into time slots. Each time slot consisted of 3600 Seconds i.e. One hour. Readings of NO2 within each time slot and location were averaged. The following Table I shows the categories of NO2 measurements in the air.

The research contributed to design an Android application that is capable to make health aware commuting recommendations to the public, based on the measured values of $\mathrm{NO} 2$ and $\mathrm{CO}$ in the city and the $\mathrm{NO} 2$ and $\mathrm{CO}$ categories available in table. In addition, the application can broadcast this information on different social media platforms.

TABLE I. AQ RANGES FOR NO2[11]

\begin{tabular}{|l|l|}
\hline Range & Category \\
\hline$(0-50)$ & Good \\
\hline$(51-100)$ & Moderate \\
\hline$(101-150)$ & Unhealthy for Sensitive Groups \\
\hline$(151-200)$ & Unhealthy \\
\hline$(201-300)$ & Very Unhealthy \\
\hline
\end{tabular}

\section{RESUlTS AND DisCUSSION}

This application was available for download in Google Play store and through effective publicity. It was downloaded for more than 5000 times. Users were offered to select their user group. Users are categorized in the following groups [11].

- People with lung disease, such as asthma.

- Children and older adults.

- People who are active outdoors.

The sensor module measured the concentration of air pollutants dynamically in different areas. There were multiple copies of this module mounted on University buses which traverse throughout the city in different times of the day. This helped us to collect data from almost all populated areas of the city in different times. An intensive data cleansing was required such as to remove duplicate and incorrect values at times. The results taken from different geographic positions (locations) are shown below in graphs.

The results shown in Fig. 3 to Fig. 17 reflects substantial variation in concentration of $\mathrm{CO}$ and $\mathrm{NO} 2$.

This research considered five routes in the city and buses commuted to these routes every hour from 7:00am to 7:00pm. Based on the reading of $\mathrm{CO}$ and NO2. For each route the Air quality index (AQI) was calculated. Results of these routes are shown in Fig. 18. From the results, it can be observed that during peak hours $8: 00 \mathrm{am}$ to $10: 00 \mathrm{am}$ and $4: 00 \mathrm{pm}$ to $6: 00 \mathrm{pm}$ the concentration of NO2 remains in the unhealthy range. Whereas during off peak times, there are opportunities for people here they can travel on these routes. This application considering health issues maps and recommends its users to commute through these routes. It is highly recommended for people with lungs diseases and children to not commute through areas with high concentration of NO2 in the air.

Based on the value of $\mathrm{CO}$ and $\mathrm{NO} 2$ concentration at particular times, this Recommender made one of the following four recommendation:

1) Commute at selected time slot (Safe)

2) Do not commute at selected time slot

3) Take alternate route

4) Do not commute at any time slot any route.

The availability of this application to the general public along with information related to the association of concentration of $\mathrm{CO}$ and $\mathrm{NO} 2$ with potential diseases helps citizen to take travel decisions in these areas throughout the day.

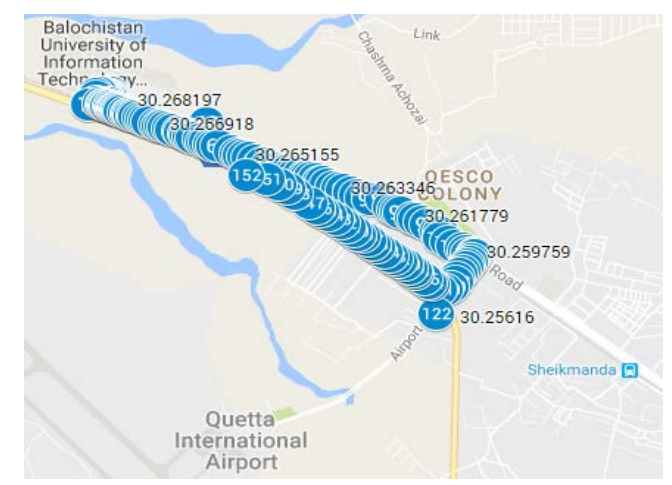

Fig. 3. Bus Route-A Location of Data Readings. 
Time

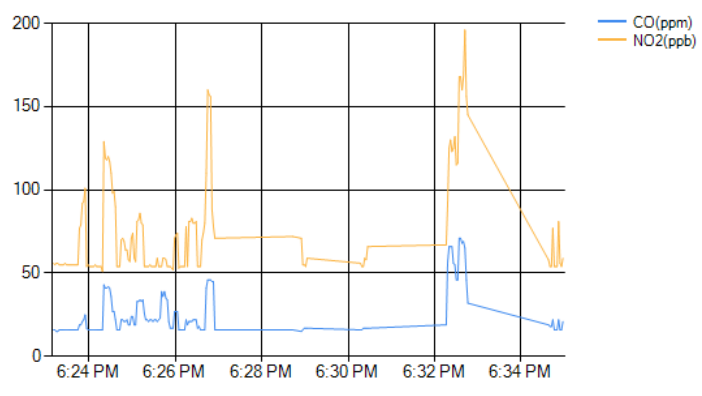

Fig. 4. Concentration Level Graph of CO (ppm) \& NO2 (ppb) Verse Time, Measured by Hardware Deployed in Vehicles.

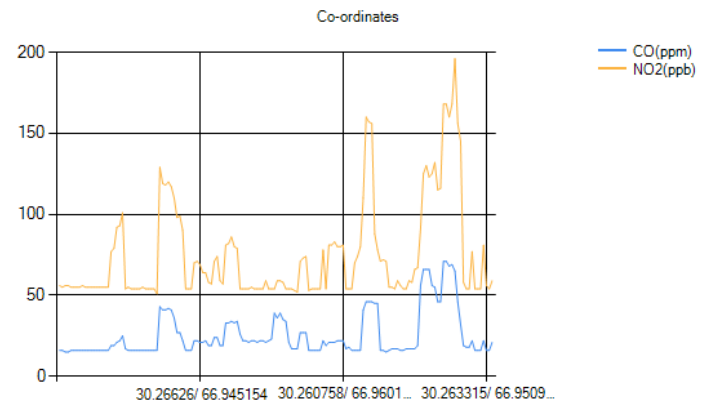

Fig. 5. CO \& NO2 Values Verses GPS Co-Ordinates, Measured by Hardware Deployed in Vehicles.

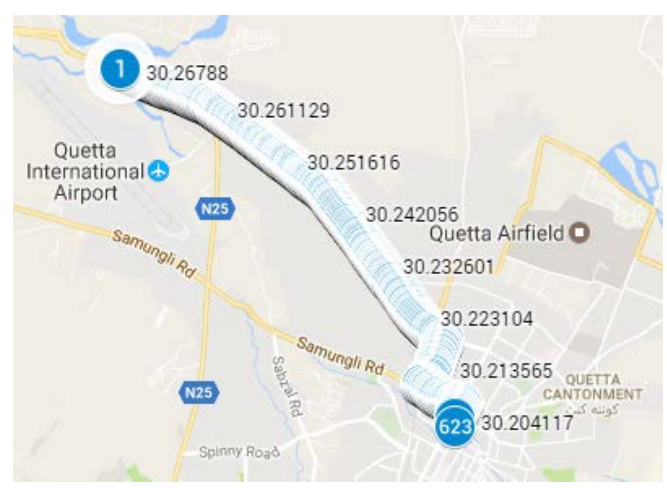

Fig. 6. Bus Route-B Location of Data Readings.

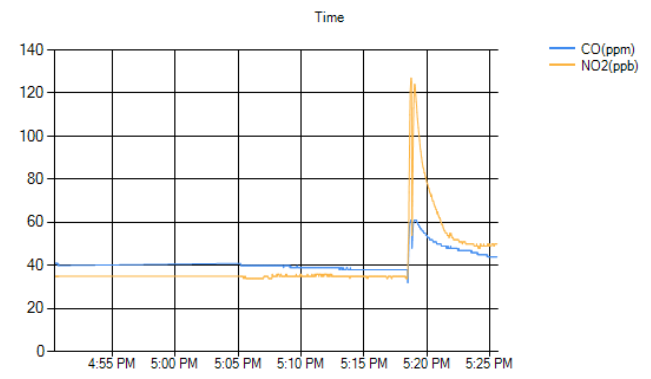

Fig. 7. CO \& NO2 Values Verses GPS Co-Ordinates, Measured by Hardware Deployed in Vehicles.

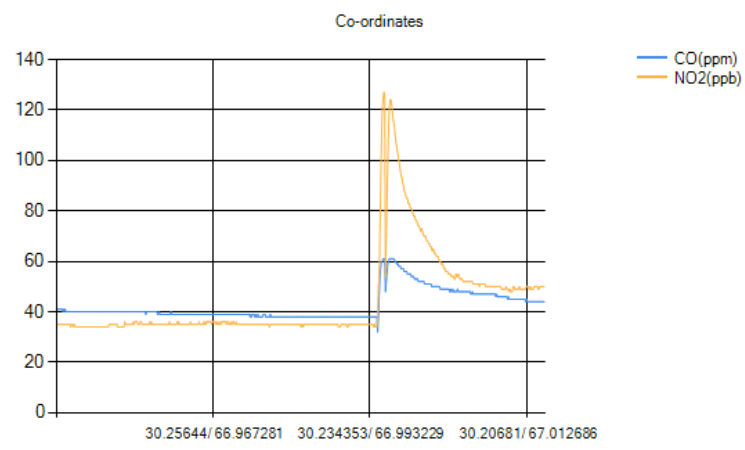

Fig. 8. CO \& NO2 Values Verses GPS Co-Ordinates, Measured by Hardware Deployed in Vehicles.

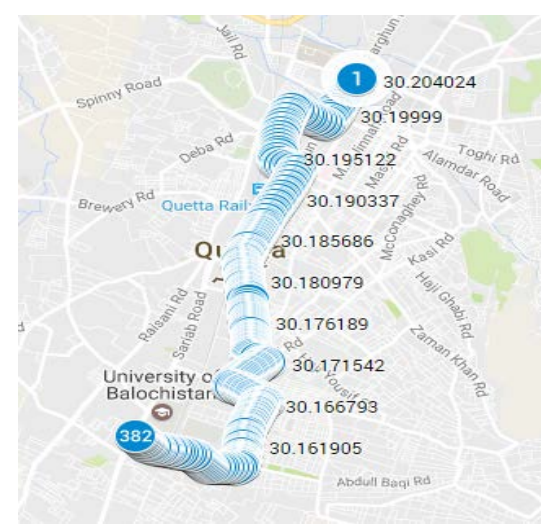

Fig. 9. Bus Route-C Location of Data Readings.

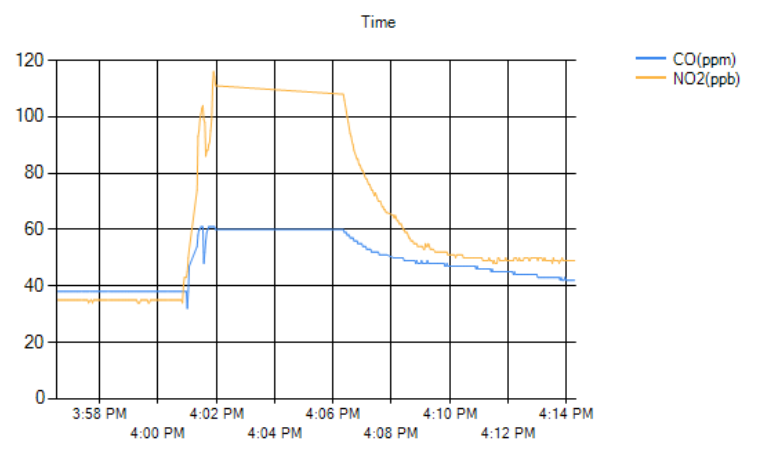

Fig. 10. CO \& NO2 Values Verses GPS Co-Ordinates, Measured by Hardware Deployed in Vehicles.

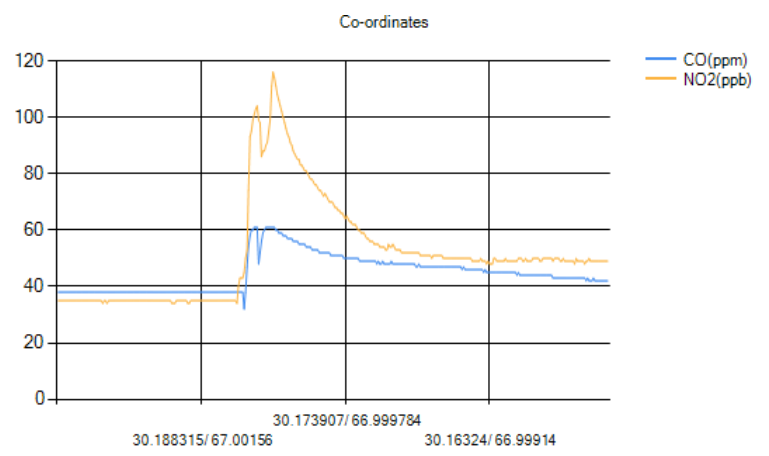

Fig. 11. CO \& NO2 Values Verses GPS Co-Ordinates, Measured by Hardware Deployed in Vehicles. 


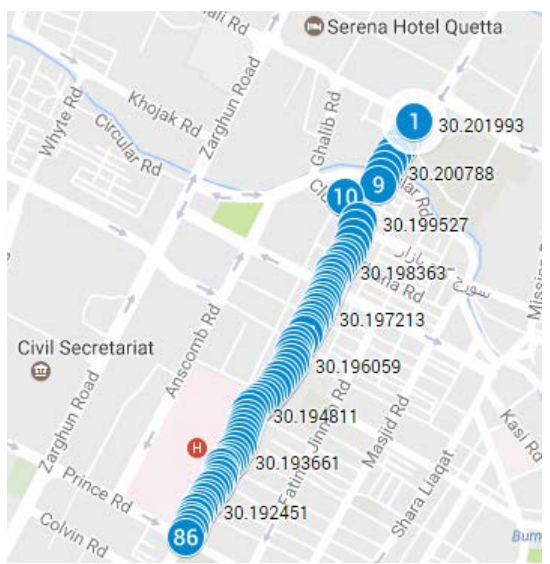

Fig. 12. Bus Route-D Location of Data Readings.

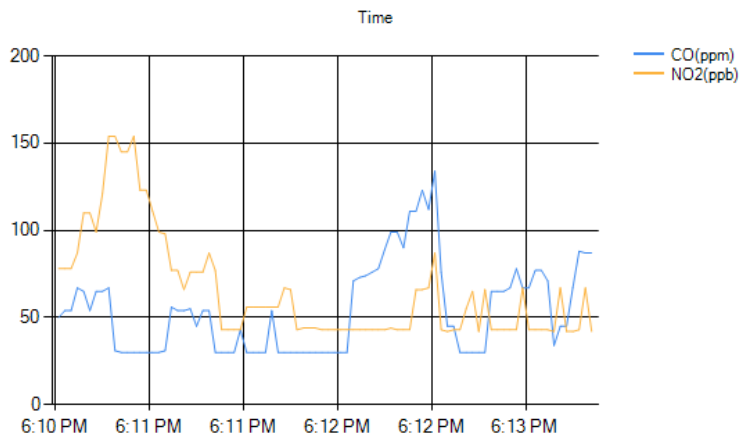

Fig. 13. CO \& NO2 Values Verses GPS Coordinates, Measured by Hardware Deployed in Vehicles.

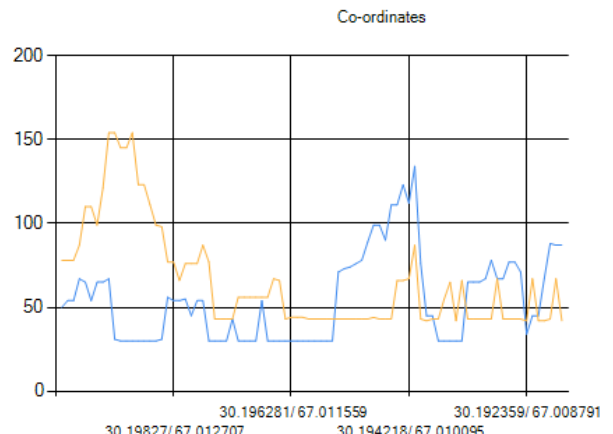

- $_{\mathrm{NO}(\mathrm{ppm})}^{\mathrm{ppb})}$

Fig. 14. CO \& NO2 Values Verses GPS Coordinates, Measured by Hardware Deployed in Vehicles.

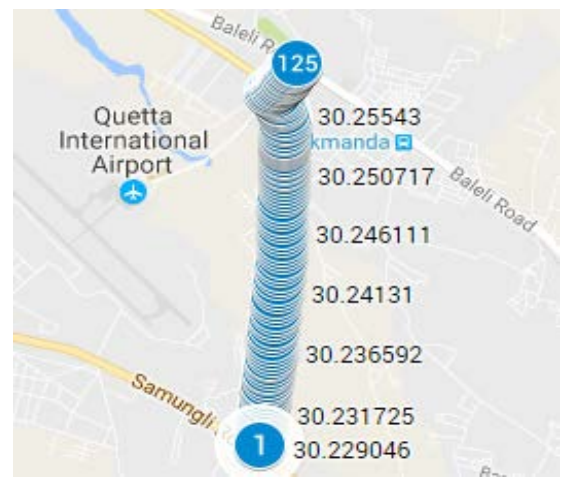

Fig. 15. Bus Route-E Location of Data Readings.

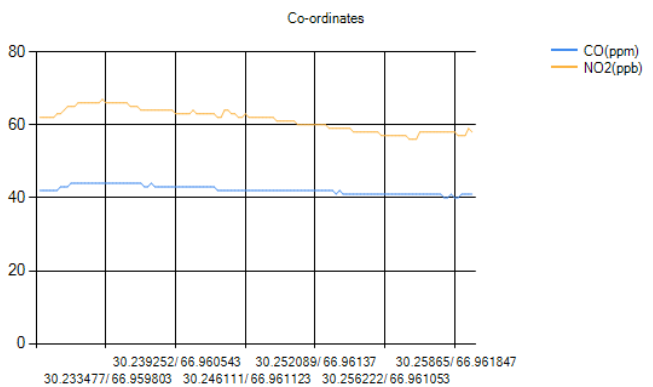

Fig. 16. CO \& NO2 Values Verses GPS Coordinates, Measured by Hardware Deployed in Vehicles.

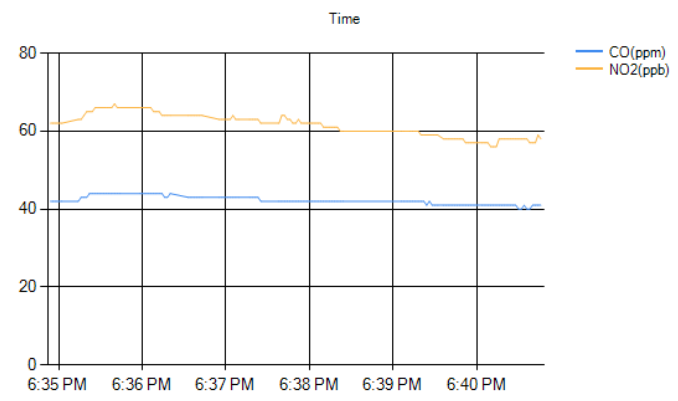

Fig. 17. CO \& NO2 Values Verses GPS Coordinates, Measured by Hardware Deployed in Vehicles.

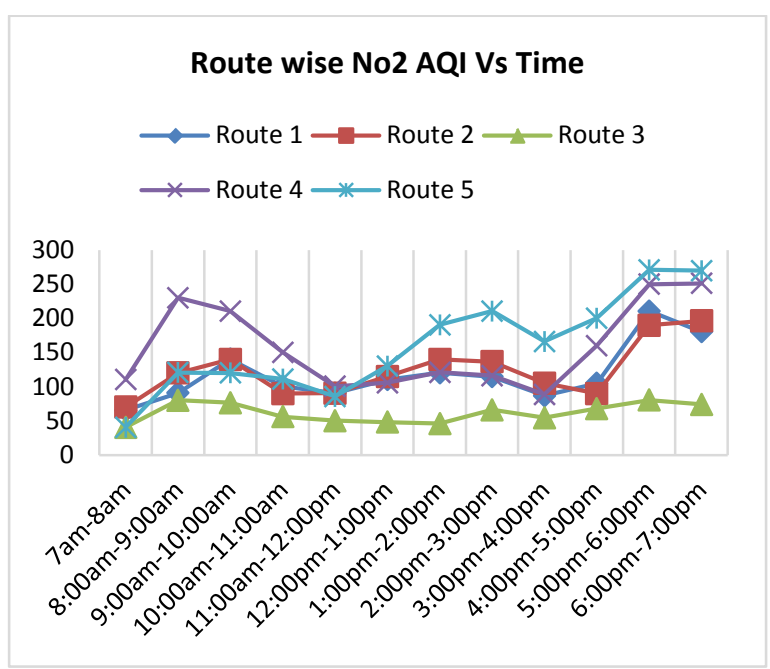

Fig. 18. Routes and NO2 AQI Against Time Slots.

\section{CONCLUSION AND FUTURE-WORK}

This research contributes health aware commuting recommendation based on cost effective monitoring of air quality. This research has presented a case study of Quetta city while dividing it in five routes and measuring AQI and making commuting recommendations through android application. It is found that as effective approach to avail the opportunity to commute in any area of interest hence there is low level of NO2. The approach may be tested and in mega city with more routes, users and multiple sensors to measure other contributing factors to AQI. The Application in future may be further integrated with multiple social media platforms to create public awareness and helping them to take timely commuting decisions. 


\section{REFERENCES}

[1] Ul-Amin R, ”Cooperative \& cost-effective network selection: a novel approach to support location-dependent \& context-aware service migration in VANETs", PhD Thesis, University of Glasgow URL: https://theses.gla.ac.uk/.

[2] R. Al-Ali, I. Zualkernan, and F. Aloul, “A mobile GPRS-sensors array for air pollution monitoring,” IEEE Sens. J., vol. 10, no. 10, pp. 16661671, 2010.

[3] R. Rushikesh, C. Mohan, and R. Sivappagari, "Development of IoT based Vehicular Pollution,” pp. 779-783, 2015.

[4] P. Völgyesi, A. Nádas, X. Koutsoukos, and Á. Lédeczi, “Air Quality Monitoring with SensorMap,” 2008 Int. Conf. Inf. Process. Sens. Networks (ipsn 2008), pp. 529-530, 2008.

[5] S. C. Hu, Y. C. Wang, C. Y. Huang, and Y. C. Tseng, “A vehicular wireless sensor network for CO2 monitoring,” Proc. IEEE Sensors, pp. 1498-1501, 2009.
[6] S. R. W. H.Ali, J.k. Soe, “A Real-Time Ambient Air Quality Monitoring Wireless Sensor Network for Schools in Smart Cities,” pp. 5-10.

[7] A.-C. Firculescu and D. S. Tudose, "Low-Cost Air Quality System for Urban Area Monitoring,” 2015 20th Int. Conf. Control Syst. Comput. Sci., pp. 240-247, 2015.

[8] D. Hasenfratz, O. Saukh, S. Sturzenegger, and L. Thiele, "Participatory Air Pollution Monitoring Using Smartphones,” 2nd Int. Work. Mob. Sens., pp. 1-5, 2012.

[9] G. V. Bhagwan and V. G. Puranik, "Real Time Air Pollution Monitoring Using Mobile Phone,” vol. 3, no. 4, pp. 447-453, 2014.

[10] S. Devarakonda, P. Sevusu, H. Liu, R. Liu, L. Iftode, and B. Nath, "Real-time air quality monitoring through mobile sensing in metropolitan areas,” Proc. 2nd ACM SIGKDD Int. Work. Urban Comput. - UrbComp ’13, p. 1, 2013.

[11] Air Quality Guide for Nitrogen Dioxide, URL: https://www3.epa.gov /airnow/NO2.pdf. 